\title{
Capping Ligand Effect On Charge Transfer Mechanism Of Hybrid Organic(P3HT):Inorganic(PbSe) Nanocomposites
}

\author{
Aarti Mehta ${ }^{1}$, Kanchan Sharma ${ }^{1,2}$, Shailesh N. Sharma ${ }^{1}$ and S. Chand ${ }^{1}$ \\ ${ }^{1}$ National Physical Laboratory, Dr. K.S.Krishnan Road, New Delhi, 110012, India \\ ${ }^{2}$ Department of Physics, Delhi Technological University, New Delhi \\ E-mail: shailesh@mail.nplindia.ernet.in, shailesh@nplindia.org
}

\begin{abstract}
In this work, we have synthesized colloidal PbSe QD's by using two different Capping ligand Oleylamine and Oleic acid. The effective diameters of PbSe QD's are in range of 6-9 nm. As-synthesized QD's are characterized by X-ray diffraction to check the phase formation while its optical properties are monitored by UV-Vis spectrometer and Photoluminescence. Inorganic PbSe QD's are dispersed in Poly (3-hexyl thiophene) polymer to make PbSe (OLA): P3HT and PbSe (OA):P3HT nanocomposites respectively. Photo stability is checked by laser induced degradation and PL Quenching experiment shows enhancement in charge transfer on varying the ligands. We found that, Oleylamine capped PbSe QD's shows higher PL quenching and hence higher Stern volmer quenching Constant $\left(K_{s v}\right)$ on account of its enhanced interaction with P3HT as compared to Oleic acid capped PbSe QD's. This shows the effective role of capping ligands particularly Oleylamine (OLA) in enhancing the charge transfer mechanism for its potential role in Organic photovoltaics.
\end{abstract}

\section{INTRODUCTION}

$\mathrm{Q}$ uantum dots (QD's) of semiconductor materials have attracted much attention because their physical and chemical properties can be systematically tuned by variation of size $[1,2]$. For over last two decades, semiconductor colloidal Quantum dots have been studied experimentally and theoretically. The attention is owed not only to academic interest in a system which wonderfully demonstrates quantum mechanical effects and lies in a realm between bulk and atomic properties, but also because of its possible application[3,4].

Colloidal II-VI and III-V semiconductor Quantum dots (QD's) are potential candidate for a wide range of applications due to their different optical and electronic properties. The exciting potential of these quantum confined materials arises from the fact that it is possible to fabricate structure of radius $\mathrm{R}$ smaller than the exciton bohr radius $\mathrm{a}_{\mathrm{B}}$. QDs of IV-VI materials such as $\mathrm{PbS}$ and $\mathrm{PbSe}$ offer unique access to the regime of extreme quantum confinement since the electron, hole and exciton all have comparatively large radii [5]. In PbSe the electron, hole and exciton radii are 23, 23 and $46 \mathrm{~nm}$, respectively. The exciton radii in PbSe is near about eight times larger than CdSe with a narrow direct bandgap $(0.28 \mathrm{ev}$ at $300 \mathrm{~K})$. Due to this it has great potential for application in NIR laser, solar cells, telecommunication etc [6]. These large radii allow strong confinement to be achieved relatively in large structures. Thus QDs IV-VI materials have properties reflecting all the benefits of strong quantum confinement, which reduces influence from surface effects; for the same level of confinement as that of QDs of II-VI or III-V materials. Furthermore the larger Bohr radii also allow for materials to be fabricated with much stronger Quantum confinement than is possible with II-VI and III-V materials [7].

Recently, highly close packed QDs in films have demonstrated amplification of their spontaneous emission in near infrared (NIR) wavelengths [4, 5]. It is reported in recent papers that $\mathrm{PbSe}$ nanocrystal QDs can efficiently produce multiple electron-hole pairs (excitons) in response of single absorbed photon. This concept contributes significantly to improve the efficiency of Solar cell [8].

The influence of nanocrystal surface property is valuable to determine the nanocrystal properties. The preparation of semiconductor nanocrystal by colloidal route usually involves capping of the nanocrystal surface with stabilizing ligands which fulfill various functions, including the passivation of dangling bonds at the surfaces. In addition, capping ligand helps to control the grain growth of the nanocrystals during its formation an increase the dispersability of nanocrystals, which can be used for different applications $[9,10]$.

In this work, we have observed that surface properties are easily modulated by ligand variation which strongly influenced their optical as well as morphological properties.

\section{EXPERIMENTAL}

PbSe QDs are synthesized by chemical route method described by Murray et al [11]. Experiments are carried out under an Argon gas inert atmosphere using schlenk type apparatus. Basically this is a hot injection method of synthesis. In a typical synthesis of PbSe nanocrystals lead acetate $(1.5 \mathrm{mg})$ in $(5 \mathrm{ml})$ of Oleic acid and Oleylamine was heated at a temperature of $100-130{ }^{\circ} \mathrm{C}$ for $2-3 \mathrm{~h}$ followed by addition of TOPSe at room temperature. The reaction mixture was stirred at a temperature between $80^{\circ} \mathrm{C}$ and $160^{\circ} \mathrm{C}$ for 1-3 hours to obtain a black suspension. The residue was washed with methanol and hexane to remove impurities and excess of ligand from surface of assynthesized QDs. 


\section{A. Required Chemicals}

Lead Acetate, Phenyl Ether, Oleic Acid, Oleylamine, Selenium metal powder and n-trioctyl phosphine (TOP) were purchased from Aldrich Chemical Co.

\section{B. Characterization}

Transmission Electron Microscopy analysis for the estimation of size, morphology and crystalline plane is carried out by using a JEOL electron microscope (JEM 2000EX). Optical spectroscopic measurements are carried out in the absorption mode using a Shimadzu UV-3101 PC spectrometer.

\section{RESULT AND DISCUSSION}

Lead selenide QDs are synthesized by varying the capping of two different ligand oleic acid and oleylamine. As synthesized PbSe QDs are washed 3-4 times with methanol and finally with hexane to remove excess of ligand from the surface of PbSe QDs. As-synthesized PbSe QDs are of sizes 6 to $9 \mathrm{~nm}$ and characterized by various techniques to check their phase formation, morphology and optical properties.
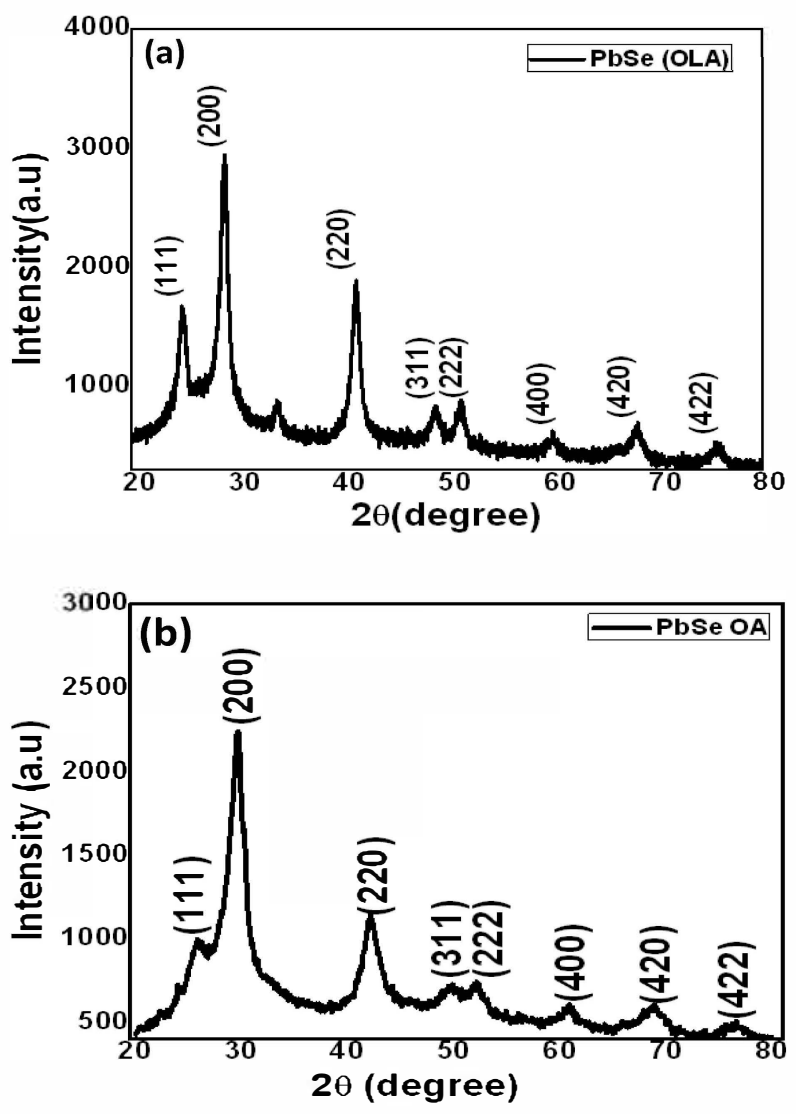

Fig .1. XRD Spectra of: (a) Oleylamine and (b) Oleic acid capped PbSe QDs.

Fig.1 (a and b) shows XRD pattern for oleylamine (OLA) and oleic acid (OA) capped PbSe QDs respectively. X-ray diffraction was performed at room temperature on a Rigaku Miniflex-II with $\mathrm{Cu} \mathrm{K} \alpha$ radiation.

Samples were prepared by drop casting method of the $\mathrm{PbSe}$ nanocrystals on glass substrate. The X-ray diffraction exhibits clear diffraction peaks at (111), (200), (220), (311) and (400) plane correspond to PbSe that confirm cubic rock salt crystal structure [5] and are in well agreement with the powder diffraction of standard PbSe. The average crystallite size is estimated by using the scherrer's equation are found to be $39.7 \mathrm{~nm}$ and $26.4 \mathrm{~nm}$ for OLA and OA capped PbSe QDs respectively, which is according with trend shown by TEM data.

The effect of capping ligand on morphology of two different QDs is studied by TEM. Fig.2 (a) and (b) shows TEM images of oleylamine and oleic acid capped PbSe QDs respectively. It is clear from the images that synthesized $\mathrm{PbSe}$ nanocrystals are spherical in shape with the size range 6 to $9 \mathrm{~nm}$ for PbSe (OA/OLA) and distributed uniformly. Agglomeration effect are present in OA capped PbSe QDs shown in fig. (b).
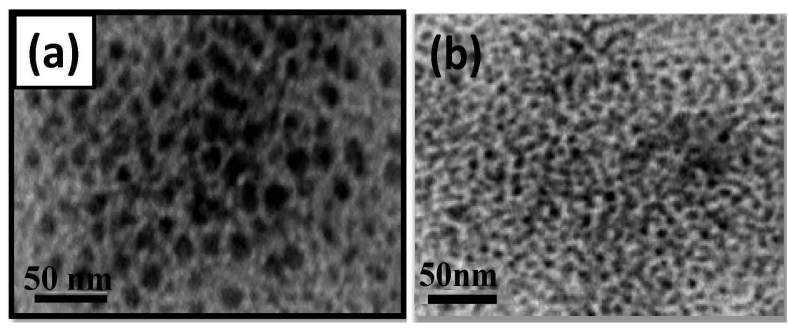

Fig .2. TEM images of PbSe QDs: (a) Oleylamine and (b) Oleic acid Capped nanocrystal.

UV- Vis absorbance spectra of as-synthesized (OLA/OA) capped PbSe QDs are shown in fig.3 (a) and (b). The absorption peak for oleylamine and oleic acid capped $\mathrm{PbSe}$ QDs are at $1744 \mathrm{~nm}, 1746 \mathrm{~nm}$ respectively. Bandgap $\mathrm{E}_{\mathrm{g}}$ calculated from absorbance spectra is in the range of 0.69 $0.70 \mathrm{eV}$ relative to the bandgap of $0.27 \mathrm{eV}$ of bulk $\mathrm{PbSe}$ respectively. Increased in bandgap value of as-synthesized $\mathrm{PbSe}$ QDs from the bulk gives the evidence of particle formation in the nanorange.
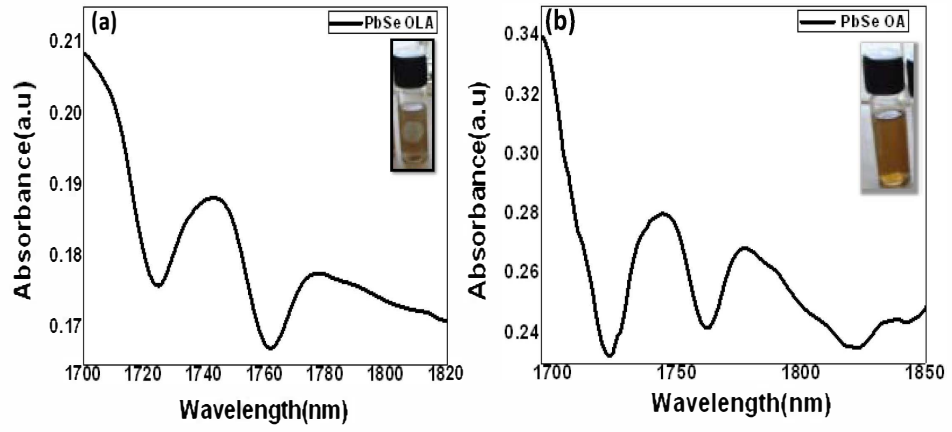

Fig .3. UV-VIS absorbance spectra of (a) Oleylamine and (b) Oleic acid Capped PbSe QDs. 
The inset in fig (a) and (b) shows the photos of OLA capped and OA capped PbSe QDs in ambient conditions. OLA capped $\mathrm{PbSe}$ QDs shows slightly gray color, whereas oleic acid capped shows brownish color.

After confirmation of the Phase formation and particle size, photoluminescence quenching experiment has been done to check the effect of capping ligand on charge transfer property from P3HT polymer to PbSe nanocrystal. fig.4. (a) and (b) shows the PL quenching curves at different concentration for P3HT: PbSe (OLA) and P3HT: PbSe (OA) nanocomposites respectively.
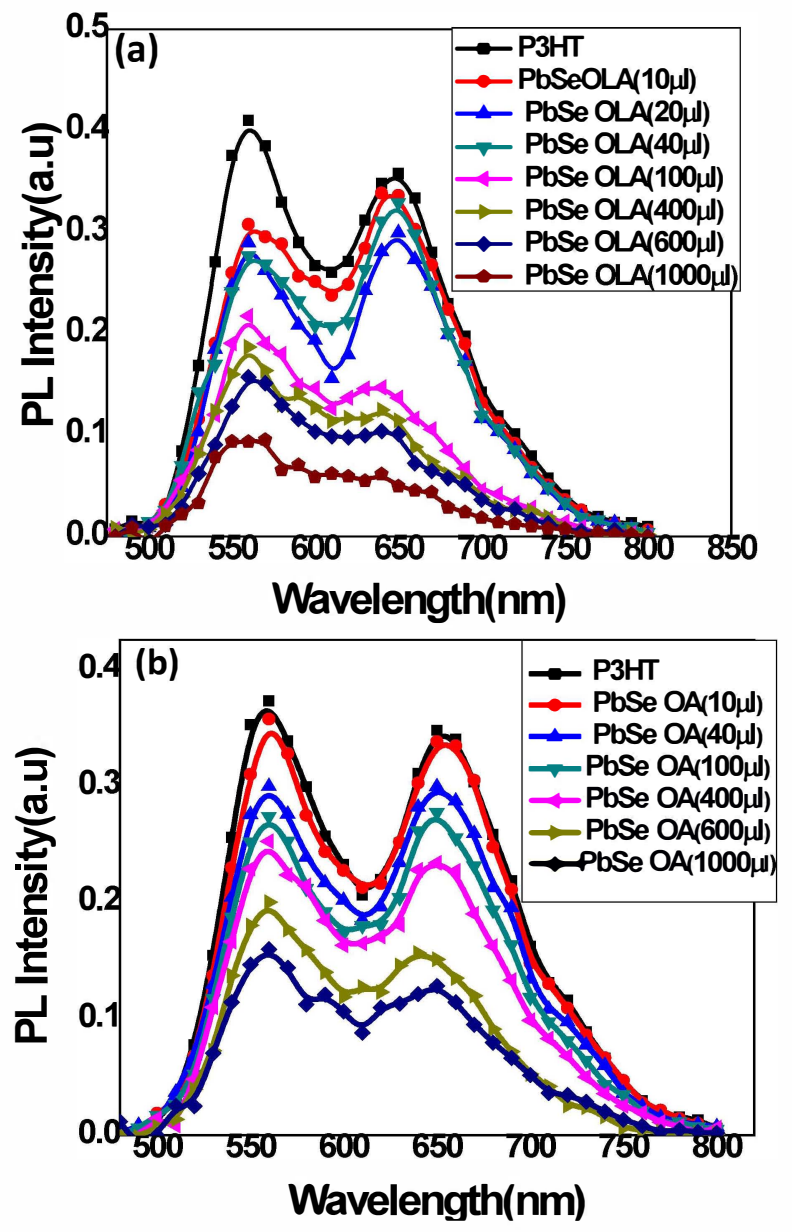

Fig .4. Emission Intensity Profile P3HT: PbSe nanocomposites at different concentrations of $\mathrm{PbSe}$ (a) P3HT: $\mathrm{PbSe}(\mathrm{OLA})$ and (b) P3HT: $\mathrm{PbSe}(\mathrm{OA})$ nanocomposites.

It is clear from the figure that significant decrease in PL intensity (PL quenching) of P3HT polymer is observed upon addition of PbSe (OLA) QDs as compared to PbSe (OA) capped QDs, indicating better charge transfer for the former as compared to the later. Change in rate of charge transfer by varying the capping ligand in $\mathrm{P} 3 \mathrm{Ht}$ : $\mathrm{PbSe}$ nanocomposite is further confirmed by PL quenching efficiency with $\mathrm{PbSe}$ concentration and Stern-Volmer plots.

Fig. 5. (a) and (b) shows the emission intensity profile of P3HT: PbSe nanocomposites as a function of $\mathrm{PbSe}$ concentration for both PbSe QDs. As evident from the fig.5 (a and $b$ ), that rate of PL decays $\Delta \mathrm{P}_{\mathrm{L}} / \mathrm{P}_{\mathrm{Li}}$ for $\mathrm{P} 3 \mathrm{HT}$ : PbSe (OLA) is 0.77 is better as compared to $\mathrm{P} 3 \mathrm{HT}$ : $\mathrm{PbSe}(\mathrm{OA})$ for which $\Delta \mathrm{P}_{\mathrm{L}} / \mathrm{P}_{\mathrm{Li}}$ is 0.57 .
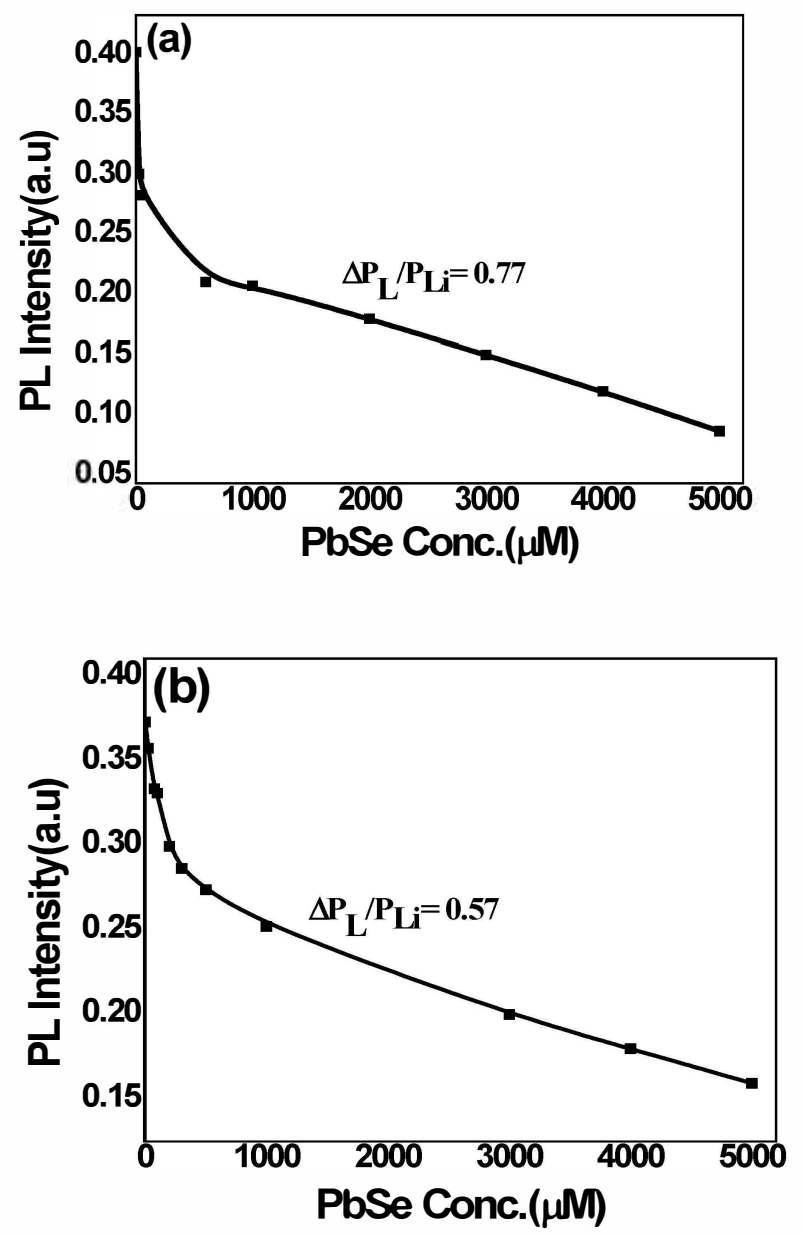

Fig .5. Variation of PL peak Intensity of polymer with PbSe QDs concentration for (a) P3HT: $\mathrm{PbSe}(\mathrm{OLA})$ and (b) P3HT: $\mathrm{PbSe}(\mathrm{OA})$ nanocomposites.

PL Intensity decay of P3Ht polymer with oleylamine capped PbSe QDs is maximum, which shows better charge transfer whereas both capping ligand having almost same chain length. It is due to efficient removal of oleylamine capping ligand from the PbSe surface compared with OA-ligand facilitates efficient charge transfer between donor and acceptor leading to the separation of photoexcited bound electron-hole pairs in the polymer and hence efficient quenching of PL emission intensity.

Capping ligand used during synthesis to control the growth of nanocrystals are present on the surface creates hindrance in interaction between polymer and QDs. So it is required to remove the excess of ligand present on the surface of QDs by some chemical treatment. As-synthesized PbSe nanocrystals are washed continuously with methanol. Due to successive washing treatment sufficient oleylamine is removed from the surface of $\mathrm{PbSe}$ as compared to oleic acid and correspondingly shows better charge transfer in P3HT: PbSe (OLA) nanocomposites than P3HT: PbSe (OA) 
nanocomposites. The similar effect, we obtained in our earlier work where, oleylamine capped CdSe QDs are synthesized which shows energy transfer but after giving washing treatment charge transfer is improved from polymer to QDs due to partial removal of capping ligand from the QDs surface [12].

The decrease in photoluminescence of polymer upon addition of PbSe (OLA/OA) nanocrystals is obtained due to charge transfer between polymer to PbSe nanocrystal.

There are two basic type of quenching : static and dynamic quenching. Both types require an interaction between the fluorophore and quencher. In the case of dynamic quenching, the quencher must diffuse to fluorophore during the life time of the excited state. Upon contact, the fluorophore returns to the ground state without emission of a photon. In the case of static quenching, a complex forms between the fluorophore and the quencher, and complex is nonfluorescent [13].
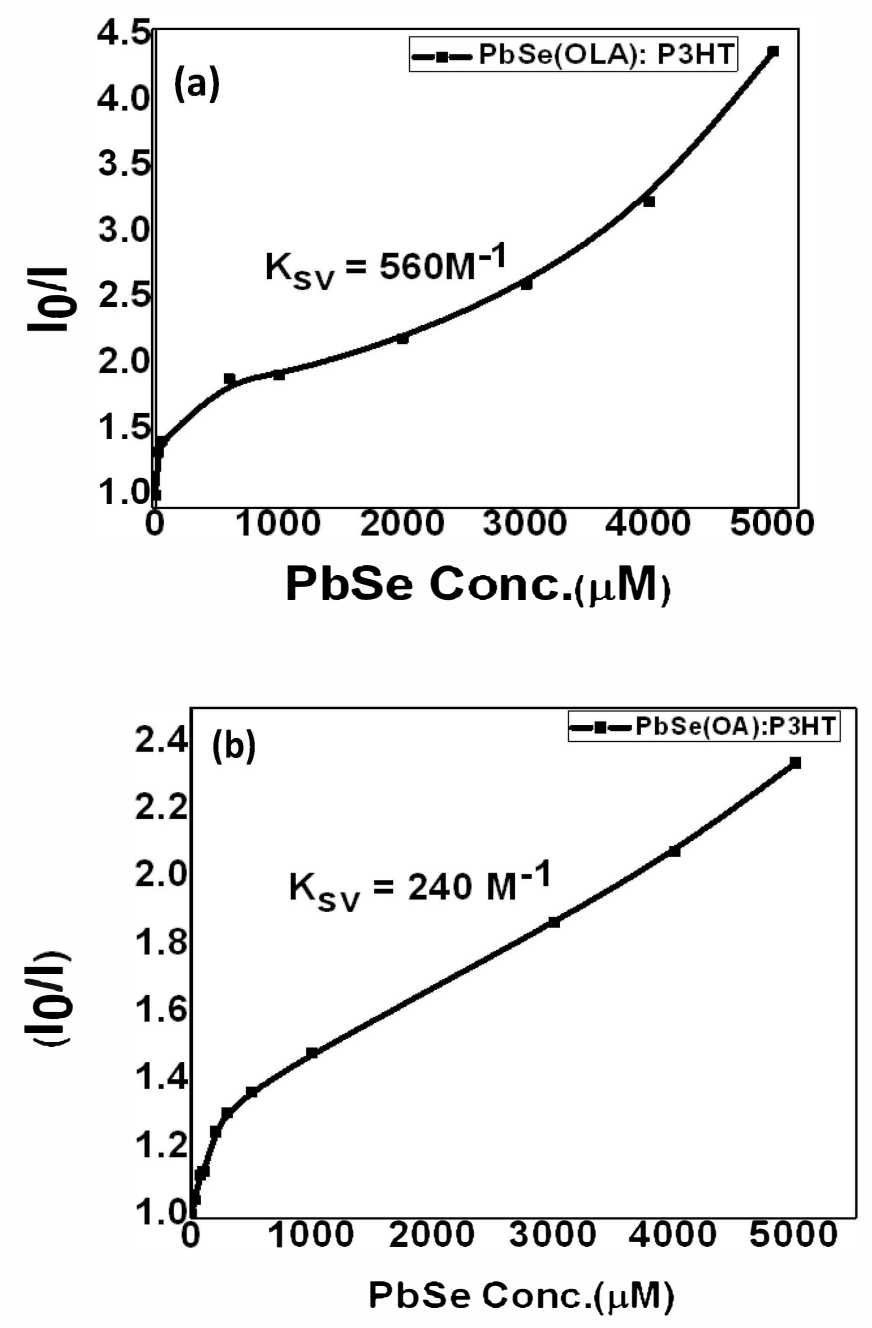

Fig .6. Stern-Volmer plots of (a) P3HT: PbSe (OLA) and (b) P3HT: $\mathrm{PbSe}(\mathrm{OA})$ nanocomposites.

In the case of collisional quenching the following relation, called stern volmer equation, holds:

$$
\left(\mathrm{I}_{0} / \mathrm{I}\right)=1+\mathrm{K}_{\mathrm{SV}}[\mathrm{Q}] \text {, }
$$

Where $\mathrm{I}_{0}$ and $\mathrm{I}$ are the fluorophore intensities in absence or presence of quencher respectively. [Q] is the quencher concentration and $\mathrm{K}_{\mathrm{sv}}$ is the stern-volmer quenching constant. A plot of $\mathrm{I}_{0} / \mathrm{I}$ versus the PbSe concentration[Q] should yield a straight line with its slope equal to $\mathrm{K}_{\mathrm{sv}}$. Such plots known as Stern -Volmer plots, are shown in fig.6. (a and b) for P3HT: PbSe (OLA) and P3HT: PbSe (OA) nanocomposites respectively. The stern volmer quenching constant are calculated for both the samples, value for $\mathrm{PbSe}(\mathrm{OLA})$ and $\mathrm{PbSe}(\mathrm{OA})$ is $560 \mathrm{M}^{-1}$ and $240 \mathrm{M}^{-1}$ respectively. The increase in value of $\mathrm{K}_{\mathrm{sv}}$ gives the significance of increase in PL Quenching and hence charge transfer in $\mathrm{PbSe}$ (OLA) QDs. Increase in value of $\mathrm{K}_{\mathrm{sv}}$ gives the evident that interation between polymer and nanocrystals is increased due to removal of capping ligand from the surface of PbSe QDs. Higher value of quenching constant indicates better charge transfer between polymer and $\mathrm{PbSe}$ (OLA) nanocomposites.

\section{CONCLUSION}

In concluding section, it has been demonstrated that $\mathrm{PbSe}$ QD's in size range 6-9nm capped with different ligand (OLA, OA) are successfully synthesized by colloidal route. PbSe QDs exhibits strong quantum confinement due their small particle sizes. Although both OLA and OA ligand act as a perfect surface passivants but easy removal of OLA ligand upon successive washing in methanol and hexane rather than toxic and harmful pyridine mixture makes it viable for the synthesis of quantized hybrid polymer: $\mathrm{PbSe}$ nanocomposites for photovoltaic application.

\section{ACKNOWLEDGEMENT}

The authors are grateful to Director, NPL for providing the facilities for the successful completion of this research work. One of the authors Aarti Mehta is thankful to MNRE for Junior Research Fellowship. I also, would like to acknowledge Dr. Jasveer Singh, IARI PUSA, New Delhi for TEM characterization.

\section{REFERENCES}

[1] Ekimov A I, Efros AI L and Onushchenko A A 1985 Solid State Commun. 56921

[2] Zunger A 1998 MRS Bull. 23 (2).

[3] Brian L. Wehrenberg, Congjun Wang, and Philippe Guyot-Sionnest, Interband and Intraband Optical Studies of PbSe Colloidal Quantum Dots J. Phys. Chem. B 2002, 106, 10634-10640

[4] Hui Du, Chialing Chen, Rishikesh Krishnan, Todd D. Krauss, Optical Properties of Colloidal PbSeNanocrystals, Nano letters 2002,vol2 no. $11,1321-1324$

[5] Efros, A. L.; Efros, A. L. So . Phys.sSemiconductors 1982, 16, 772.

[6] Umesh Kumar, shailesh N. Sharma, sukhvir Singh, M. Kar, V.N.Singh, B.R.Mehta, Rita Kakkar Material Chem. Phys. 113 (2009) 107-114

[7] Wise, F. W. Acc. Chem. Res. 2000, 33, 773

[8] Richard D. Schaller, Melissa A. Petruska and Victor I.Klimov 2005 Applied Phys Letters 87, 253102

[9] M. J. Fernee, E. Thomsen, P. Jensen, H.Rubinsztein-Dunlop, Nanotechnology. 17 (2006) 956. 
[10] I.C. Baek, S. I. Seok, N.C. Pramanik, S. Jana, M.A.Lim, B.Y. Ahn., C.J. Lee, Y.J.Jeong, J. Colloid \& Interface Sci. 310(1) (2007) 163.

[11] C B Murray, S. Syun, W. Gaschler, H. Doyle, T.A. Betley, C R Kagan, IBM J. Res. Dev. 45 (2001) 47.

[12] Aarti Mehta, Shailesh N. Sharma, V.N. Singh, A.K. Srivastva and S. Chand, Enhancement in Charge Transfer Mechanism by Non-LigandExchange Process for Colloidal Hybrid Organic (MEHPPV):Inorganic (CdSe) Nanocomposites, accepted in SPIE proceedings.

[13] Kusum kumara, Umesh kumar, Shailesh N Sharma, Suresh Chand, J.Phys.D: Appl Phys, 41 (2008) 235409(9PP). 